\title{
OPINIÃO DOS ALUNOS QUANTO AO ENSINO DOS DIAGNÓSTICOS DE ENFERMAGEM SEGUNDO A TAXONOMIA DA NANDA
}

\author{
Maria Helena Baena de Moraes Lopes* \\ Ianê Nogueira do Vale** \\ Margarida Barbosa***
}

LOPES, M. H. B. de M. et al. Opinião dos alunos quanto ao ensino dos diagnósticos de enfermagem segundo a Taxonomia da NANDA. Rev. Esc. Enf. USP, v.31, n.1, p.80-8, abr. 1997.

Acreditando na importância do diagnóstico de enfermagem,o seu ensino foi introduzido há 4 anos nas disciplinas de Assistência de Enfermagem à Saúde da mulher e Enfermagem Perinatal, no 6o. período do curso de graduação em enfermagem da UNICAMP.Com o intuito de avaliar essa experiência, propusémo-nos a levantar a opinião dos alunos e obter subsidios para aprimorar o ensino. Foram ministradas 4 horas de ensino teórico e indicada bibliografia básica. A aplicação prática supervisionada foi desenvolvida durante 21 horas numa unidade neonatal e 45 horas em centros de saúde. Ao término das disciplinas, 16 alunos responderam a um questionário com questões fechadas e abertas. O grupo de alunos respondentes foi unânime em considerar válido o estudo dos diagnósticos de enfermagem. A maioria considerou mais adequado introduzir o ensino no 3o. semestre, quando se iniciam os procedimentos básicos de enfermagem. Quanto à dificuldade de aprendizado do uso dos diagnósticos, $68,7 \%$ dos alunos relataram um nível de dificuldade superior a $25 \%$ e inferior a 75\%. O grupo de alunos como um todo achou que o estudo do diagnóstico de enfermagem trouxe modificações no seu conceito de assistência de enfermagem.

UNITERMOS: Diagnóstico de enfermagem. Ensino

\section{INTRODUÇÃO}

O diagnóstico de enfermagem foi introduzido no Brasil em 1967 por Horta que se baseou na teoria da motivação humana de Maslow. Horta propôs uma assistência de enfermagem sistematizada em seis fases, sendo o diagnóstico uma

\footnotetext{
* Profa. Dra. do Depto de Enfermagem - FCM - UNICAMP e Coordenadora do Programa de Educação Continuada - CAISM - UNICAMP

** Profa. Assistente do Depto. de Enfermagem - FCM - UNICAMP e Diretora da Divisão de Enfermagem - CAISM - UNICAMP

*** Enfermeira da Divisão de Enfermagem - CAISM - UNICAMP
} 
dessas fases (HORTA, 1979).Posteriormente a tendência de alguns serviços de enfermagem foi simplificá-lo em três fases (histórico, prescrição de enfermagem e evolução) e substituir o termo diagnóstico de enfermagem por problemas de enfermagem. A prática de listar problemas expressando os sinais e sintomas de uma doença, a própria doença, os procedimentos terapêuticos e o uso de equipamentos, com o objetivo de identificar as necessidades de atenção e cuidados ao paciente, substituindo o diagnóstico de enfermagem, é discutível e tem gerado dúvidas sobretudo com respeito à definição do que são problemas de enfermagem (MARIA; ARCURI, 1991).

Na opinião de FRIEDLANDER (1977) são raros os problemas de enfermagem e explica desta forma: " o que existe são problemas do paciente em geral, tão complexos que sua solução so é possivel mediante a atuação de vários profissionais. Não só a marcante especialização dos profissionais, como a variedade de aspectos oferecidos por um só problema, faz-nos acreditar que cada problema do paciente tem apenas alguns aspectos nos quais a enfermeira atua. Nesse caso não o podemos denominar problema de enfermagem".

$O$ termo diagnóstico ficou esquecido pela enfermagem brasileira, reaparecendo na literatura a partir de 1988/1989, após uma lacuna de quase 20 anos (MARIA; ARCURI, 1991).

HORTA em 1977, observou que na aplicação do processo de enfermagem o enfermeiro encontra uma grande dificuldade para estabelecer o diagnóstico. Atribuiu como causas: o desconhecimento de sintomas (problemas de enfermagem), de necessidades básicas alteradas e da nomenclatura destas necessidades, entre outras.

A NANDA (North American Nursing Diagnosis Association) em 1990 definiu o diagnóstico de enfermagem como sendo um julgamento clínico das respostas do indivíduo ou da família ou da comunidade aos processos vitais ou aos problemas de saúde atuais ou potenciais, os quais fornecem a base para a seleção das intervenções de enfermagem, para atingir resultados pelos quais o enfermeiro é responsável (FARIAS et al.,1990). Portanto, o conceito de diagnóstico de enfermagem transcende o de problema de enfermagem não podendo ser usado como um sinônimo.

O diagnóstico de enfermagem é importante pelas seguintes razões, entre outras:

1. identifica os problemas do cliente que estão no âmbito de resolução da enfermagem;

2. cria um linguagem própria da enfermagem;

3. identifica lacunas sobre as intervenções de enfermagem.

Acreditando na importância do diagnóstico de enfermagem e na necessidade de se utilizar uma linguagem mais universal, nós, docentes das disciplinas de Assistência de Enfermagem à Saúde da Mulher e Enfermagem Perinatal, desde 1993 temos introduzido o ensino dos diagnósticos segundo a Taxonomia I revisada da NANDA (MESSIAS, 1991). 
Até o momento não havíamos feito nenhuma avaliação do ensino dos diagnósticos de enfermagem e numa primeira etapa nos propusemos a levantar a opinião dos alunos e assim, obter subsidios para aprimorar o ensino a fim de melhor capacitá-los a utilizar a taxonomia NANDA dentro do processo de enfermagem.

\section{OBJETIVOS}

\section{GERAL}

Conhecer a opinião dos alunos de graduação de enfermagem quanto ao ensino do diagnóstico de enfermagem segundo a taxonomia da NANDA nas disciplinas de Assistência de Enfermagem à Saúde da Mulher e de Enfermagem Perinatal.

\section{ESPECÍFICOS}

- verificar a opinião dos alunos quanto a validade do ensino do diagnostico de enfermagem nas disciplinas.

- identificar qual período do curso de enfermagem seria o mais adequado para introduzir o estudo sobre o diagnóstico de enfermagem, na opinião dos alunos.

- conhecer o nível de dificuldade sentido pelos alunos durante a aplicação prática do diagnóstico de enfermagem.

- verificar se, na opinão dos alunos, o estudo do diagnóstico de enfermagem trouxe modificações no seu conceito de assistência de enfermagem.

\section{METODOLOGIA}

O curso de graduação de enfermagem do Departamento de Enfermagem da Faculdade de Ciências Médicas da Universidade Estadual de Campinas(UNICAMP) tem a duração de quatro anos ou oito semestres e conta com 30 vagas por turma.

O ensino do diagnóstico de enfermagem segundo a taxonomia da NANDA foi ministrado aos 20 alunos do 60 . semestre do curso de graduação em enfermagem durante o desenvolvimento das disciplinas de Assistência de Enfermagem à Saúde da Mulher e Enfermagem Perinatal, no 2o. semestre de 1994. 
Foram ministradas 4 horas de ensino teórico e indicada bibliografia básica para leitura e consulta. A aplicação prática foi desenvolvida durante 21 horas de estágio numa unidade neonatal, onde a assistência de enfermagem é sistematizada e se utiliza a taxonomia da NANDA, e 45 horas de estágio em centros de saúde, sob supervisão das docentes.

Os alunos fizeram apresentação escrita de três estudos de caso e dois seminários utilizando a taxonomia da NANDA. O modelo de estudo de caso utilizado incluia o processo de enfermagem com as seguintes etapas: avaliação (coleta e análise dos dados), diagnóstico de enfermagem, resultados esperados, prescrição de enfermagem e avaliação final.

Ao término das disciplinas, os alunos responderam a um questionário com questões fechadas e abertas (anexo 1). Os questionários foram respondidos em sala de aula, não sendo necessária a identificação do aluno.

Para a análise dos dados, foi verificada a freqüência das respostas às questões fechadas. Foram extraídos diferentes conceitos das respostas às questões abertas e verificadas as suas freqüências.

\section{RESULTADOS}

Foram respondidos 16 questionários. Dentre estes, em 100\% das respostas os alunos acharam válido ter estudado o Diagnóstico de Enfermagem segundo a taxonomia da NANDA nas disciplinas.

As razões apontadas pelos alunos foram que o estudo dos diagnósticos de enfermagem amplia/modifica a visão sobre a assistência de enfermagem, não é ministrado em outras disciplinas, contribue para a melhoria da qualidade da assistência e porque é uma linguagem própria da enfermagem (tabela 1).

TABELA 1 - Freqüência das justificativas apontadas pelos alunos quanto a validade do ensino dos diagnósticos de enfermagem. Campinas-1994

\begin{tabular}{lcc}
\hline \multicolumn{1}{c}{ JUSTIFICATIVA } & n & $\%$ \\
\hline $\begin{array}{l}\text { amplia/modifica a visão sobre a } \\
\text { assistência de enfermagem }\end{array}$ & 9 & 42,8 \\
$\begin{array}{l}\text { não é ministrado em outras } \\
\text { disciplinas }\end{array}$ & 3 & 14,30 \\
$\begin{array}{l}\text { contribue para a melhoria da } \\
\text { qualidade da assistência }\end{array}$ & 8 & 38,10 \\
$\begin{array}{l}\text { é uma linguagem própria de } \\
\text { enfermagem }\end{array}$ & 1 & 4,80 \\
\multicolumn{1}{c}{ TOTAL } & 21 & $\mathbf{1 0 0 , 0 0 \%}$ \\
\hline
\end{tabular}


O melhor momento para se introduzir o ensino do diagnóstico de enfermagem na opinião da maioria dos alunos $(62,5 \%)$ é o 3o. semestre quando são ministradas disciplinas da área Fundamental. Todos os alunos (100\%) consideraram que o ensino dos diagnósticos deveria acontecer antes do 60. semestre (tabela 2).

TABELA 2 - Distribuição percentual do semestre em que, na opinião dos alunos, é mais adequada a introdução do estudo dos diagnósticos de enfermagem. Campinas-1994

\begin{tabular}{ccc}
\hline SEMESTRE & $\mathbf{n}$ & $\%$ \\
\hline $1^{\circ}$ & 1 & 6,25 \\
$2^{\circ}$ & 3 & 18,75 \\
$3^{\circ}$ & 10 & 62,50 \\
$4^{\circ}$ & 1 & 6,25 \\
$5^{\circ}$ & 1 & 6,25 \\
TOTAL & 16 & $\mathbf{1 0 0 , 0 0}$ \\
\hline
\end{tabular}

Quanto ao grau de dificuldade sentido pelos alunos na utilização do diagnóstico de enfermagem, $75 \%$ apontaram um nível de dificuldade de até $50 \%$ e $25 \%$ dos alunos consideraram que o nível de dificuldade foi de 50 a $75 \%$ (tabela 3).

TABELA 3 - Distribuição percentual do nível de dificuldade encontrado, na opinião do aluno, para aplicar o diagnóstico de enfermagem

\begin{tabular}{cccc}
\hline NÍVEL DE DIFICULDADE & $\mathbf{n}$ & $\%$ \\
\hline $0-\ldots--25 \%$ & 5 & 31,30 \\
$25-\ldots-50 \%$ & 7 & 43,70 \\
$50----75 \%$ & 4 & 25,00 \\
TOTAL & $\mathbf{1 6}$ & $\mathbf{1 0 0 , 0 0}$ \\
\hline
\end{tabular}

Todos os alunos respondentes consideraram que houve modificação no seu conceito de assistência de enfermagem na prática.

As razões levantadas pelos alunos foram que o estudo do diagn6stico facilitou/modificou a assistência prestada e ofereceu parâmetros para a avaliação da assistência de enfermagem, contribuiu para a definição do papel do enfermeiro e modificou a visão do aluno em relação ao cliente (tabela 4). 
TABELA 4 - Freqüência das justificativas dos alunos quanto às modificações no seu conceito de assistência de enfermagem em decorrência do estudo dos diagnósticos de enfermagem

\begin{tabular}{lcc}
\hline \multicolumn{1}{c}{ JUSTIFICATIVA } & n & $\%$ \\
\hline $\begin{array}{l}\text { Facilitou/modificou a assistência prestada e ofereceu parâmetros para } \\
\text { a avaliação da assistência de enfermagem } \\
\text { Contribuiu para a definição do papel do enfermeiro } \\
\text { Modificou a visão em relação ao cliente } \\
\text { TOTAL }\end{array}$ & 16 & 72,70 \\
\end{tabular}

\section{DISCUSSÃO}

O grupo de alunos respondentes foi unânime em considerar válido o estudo dos diagnósticos de enfermagem. O diagnóstico abre possibilidades para 0 desenvolvimento da enfermagem porque cria uma linguagem própria para descrever os problemas do cliente que a(o) enfermeira $(0)$ tem competência para resolver. Na opinão de CRUZ (1992), a busca e organização dos conhecimentos para a proposição de ações independentes da(o) enfermeira(o) fica prejudicada quando não se tem uma terminologia que expresse o julgamento que fazemos acerca dos problemas dos clientes.

Assim, o uso de uma linguagem própria de enfermagem contribue para o desenvolvimento do seu corpo de conhecimentos além de efetivar a comunicação entre as(os) enfermeiras(os). Isso evidencia-se nas justificativas apontadas pelos alunos de que o estudo dos diagnósticos é importante porque além de ser uma linguagem própria da enfermagem, amplia/modifica a visão sobre a assistência de enfermagem e contribue para a melhoria da qualidade da assistência de enfermagem.

Quanto ao momento em que é ensinado o diagnóstico de enfermagem,a maioria dos alunos considerou mais adequado o $3^{\circ}$ semestre, quando se inicia 0 ensino dos procedimentos básicos de enfermagem. Pode-se inferir que os alunos gostariam de ter iniciado mais precocemente o estudo do diagnóstico de enfermagem a fim de aprender de forma progressiva e ter mais contato com a nova terminologia.

Para CRUZ (1992), se em cada disciplina fossem abordados os diagnósticos mais frequentes da clientela a ser assistida, juntamente com a utilização de conhecimentos e habilidades adquiridos com a aprendizagem de diagnósticos abordados em disciplinas anteriores, os alunos iriam adquirir o conhecimento de forma progressiva e cumulativa o que tornaria a sua formação mais consistente. 
Infelizmente as disciplinas de Assistência à Saúde da Mulher e Enfermagem Perinatal são as unicas a abordarem este tema no curso em questião o que certamente cria dificuldades para o aluno porque para ele esse é um assunto tão novo e complexo como são os conteúdos específicos dessas disciplinas, aliado ao fato de que não há continuidade desse estudo nos semestres subsequentes o que certamente irá dificultar a assimilação e a aquisição de habilidade no uso dos diagnósticos de enfermagem.

Essa dificuldade de aprendizado do uso dos diagnósticos de enfermagem $e$ confirmada pelo achado de que $68,7 \%$ dos alunos relataram um nível de dificuldade superior a $25 \%$. Nos estudos de caso realizados pelos alunos e nas atividades práticas, nós, docentes, observamos que as maiores dificuldades estavam na coleta de dados, muitas vezes incompleta, na análise desses dados e nas prescrições das ações de enfermagem. Os alunos geralmente construiram corretamente os enunciados dos diagnósticos mas não registraram os achados que os embasariam. Quanto às prescrições, faltou pesquisa junto a literatura pertinente e uso de conhecimento adquirido em disciplinas anteriores (de enfermagem ou não) a fim de elencar ações especificas para cada diagnóstico. " $\mathrm{O}$ processo diagnóstico, em princípio, não é um fenômeno novo para o enfermeiro. Aliás este $e$ um fenômeno comum a diversas profissões. Na realidade, a novidade (e a dificuldade) consiste em diagnosticar dentro de um referencial inteiramente de enfermagem, pois, o nosso conhecimento técnico, os nossos dados significativos, as nossas interpretações e nomeações estão ainda fortemente caldadas no modelo biomédico. Assim, é por vezes mais fácil para nós diagnosticarmos em nosso cliente uma doença do que uma resposta alterada" (CRUZ, 1991). Apesar das dificuldades sentidas pelos alunos e percebidas por nós docentes, o grupo de alunos como um todo achou que o estudo do diagnóstico de enfermagem trouxe modificações no seu conceito de assistência de enfermagem. Isso se deu primeiramente facilitando/modificando a assistência prestada e oferecendo parâmetros para a avaliação da assistência de enfermagem: "...na prática aprendi a fazer determinadas observações que eu não achava importante..."; " ...a assistência fica mais direcionada aos problemas do paciente e não só uma assistência voltada para uma doença específica..."; "...orientou-me a ordenar a prioridade da prestação dos serviços de enfermagem..."; “... mostrou a necessidade de prescrever cuidados específicos e não rotinas..."; "...eu adquiri mais parâmetros para avaliar a ação...". Também levou o aluno a modificar sua visão em relação ao cliente: "...propicia uma visão global do paciente e não o segmento em partes de acordo com a patologia que ele apresenta no momento..." Além disso contribuiu para a definição do papel do enfermeiro: “...é importante que a enfermagem se organize como classe e delimite os seus horizontes..."; "...ficou mais claro o papel da enfermagem e sua atuação...".

Esses achados estão de acordo com o parecer de SHIOTSU (1991) de que a utilização dos diagnósticos de enfermagem como parte do processo de enfermagem poderá ser um instrumento para, dentre outras coisas: aproximar e manter a(0) 
enfermeira(o) junto do cliente; assegurar a individualização e uma assistência humanizada; favorecer a interação entre profissional, cliente e familia; estimular o aprimoramento profissional; possibilitar a criação de uma linguagem comum, universalizada; permitir definir melhor as áreas de atuação; propiciar a reflexão sobre a prática de enfermagem e desenvolver o pensamento crítico e a capacidade para interpretar melhor os dados que se tem a mão. Os resultados desse estudo evidenciam portanto que embora com dificuldades e limitações o ensino dos diagnósticos de enfermagem na forma como foi desenvolvido trouxe modificações conceituais importantes para os alunos. Evidenciam também a necessidade de se iniciar esse estudo nas diciplinas de fundamentos de enfermagem continuandose o seu ensino nas demais disciplinas.

\section{CONCLUSÕES}

- os alunos na sua totalidade acharam válido o ensino dos diagnósticos de enfermagem nas disciplinas de Assistência de Enfermagem à Saúde da Mulher e de Enfermagem Perinatal;

- os alunos em sua maioria consideraram que o terceiro semestre seria o mais adequado para o ensino dos diagnósticos de enfermagem e da taxonomia da NANDA;

- o nível de dificuldade sentido por $68,7 \%$ dos alunos, na aplicação prática dos diagnósticos de enfermagem, foi maior do que $25 \%$ e menor do que $75 \%$;

- o estudo dos diagnósticos de enfermagem trouxe modificações no conceito dos alunos quanto a assistência de enfermagem facilitando e modificando a assistência prestada.

\section{REFERÊNCIAS BIBLIOGRÁFICAS}

CRUZ, D. de A.L.M. da Os diagnósticos de enfermagem no ensino e na pesquisa.

Rev.Esc.Enf.USP, v.26, n.3, p. 427.34, 1992.

CRUZ, I. C. F. da Diagnóstico de enfermagem e sua aplicaçăo: revisăo da literatura.

Rev.Esc.Enf.USP v. 24, n. 1, p. 149-62, 1990.

Considerações sobre o julgamento clínico em enfermagem. In: SIMPÓSIO

NACIONAL SOBRE DIAGNÓSTICOS DE ENFERMAGEM. 1', São Paulo, 1991. Anais. São

Paulo, GIDE, 1991. p. 155-68. 
FARIAS, J. N. de et al. Diagnóstico de enfermagem: uma abordagem conceitual e prática. João Pessoa, Santa Marta, 1990.

FRIEDLANDER, M. R. Problemas de enfermagem e sua conceituaçaro. Rev.Esc.Enf.USP, v. 11 , n. 3, p. $325-30,1977$.

HORTA, W. de A. Diagnóstico de enfermagem: representação gráfica. Enf. Novas_Dimensðes. v. 3, n. 2, p. $75 \cdot 7,1977$.

Processo de enfermagem. Sáo Paulo, EPU, 1979. p. 37 - 38: Histórico.

MARIA, V. L. R. ; ARCURI, E. A. de M. O ensino e a prática do diagnóstico de enfermagem em uma instituição governamental. In: SIMPÓSIO NACIONAL SOBRE DIAGNÓSTICOS DE ENFERMAGEM. $1^{\circ}$, São Paulo, 1991. Anais. Sáo Paulo, GIDE, 1991, p. 6-45.

MESSIAS, D. K. H. Introdução de enfermeiros e alunos ao conceito do diagnóstico de enfermagem: análise teórico-prática. In: SIMPÓSIO NACIONAL SOBRE DIAGNÓSTICOS DE ENFERMAGEM. 1ª São Paulo, 1991. Anais. São Paulo, GIDE, 1991, p. 225.

SHIOTSU, C. H. Facilidades na utilização dos diagnósticos de enfermagem. In: SIMPÓSIO NACIONAL SOBRE DIAGNÓSTICOS DE ENFERMAGEM. 1 $^{\circ}$ São Paulo, 1991. Anais. São Paulo, GIDE, 1991, p. 175-82.

LOPES, M. H. B. de M. et al. Students opinion as to the teaching of the nursing diagnosis according to NANDA. Rev. Esc. Enf. USP, v.31, n.1, p.80-8, abr. 1997.

In accordance to North American Nursing Diagnosis Association (NANDA), nursing diagnosis is a clinical judgement of the individual, family or community answers to the vital process or to the actual or potential health problems. These gives the basis for the itervention selection by which the nurse is responsible. Believing in the importance of nursing diagnosis, its teaching was introduced 4 years ago in the subjects of Woman s Health Assistence and Perinatal Nursing, in the 6o. period of the undergraduate course in nursing at UNICAMP. Aiming at evaluating these experience, we proposed to get the students opinion and obtain subsides to perfect teaching. Teoric teaching of 4 hours was given and it was indicated the basical bibliography. The supervised pratical application was developed during 21 hours in a neonatal unit and 45 hours in health centers. At the end of the subjects, 16 students aswered a form with closed and open questions. All the students considered valid the study of the nursing diagnoses. Most of the students considered more proper to introduce the teaching in the 3o. semester, when the nursing basic procedues is begun. As to the difficult of learning in the use of diagnoses, $68,7 \%$ of the students reported a diffucult level higher to $25 \%$ and lower to $75 \%$. The students group consideres that the study of nursing diagnoses brought modifications in its concept of nursing assistance.

UNITERMS: Nursing diagnosis. Teaching 